\title{
Digital Twin Approach for Solving Reconfiguration Planning Problems in RMS
}

\author{
Kezia Amanda Kurniadi, Sangil Lee and Kwangyeol Ryu \\ Pusan National University, Busan, South Korea \\ kyryuepusan.ac.kr
}

\begin{abstract}
Reconfigurable Manufacturing System (RMS) appeared as a solution to high variation in customer demands allowing manufacturers to satisfy different amount of demands in different period. RMS satisfies demands by rapidly reconfiguring its hardware and software components, in order to quickly adjust its production capacity and functionality within a part family in response to sudden market changes or intrinsic system change depends on the demand of every single period. This reconfiguration process brings a critical issue within the RMS that is called as reconfiguration planning problems (RPP) introduced in this paper. With the rise of digital twin that has been a global issue, many companies or manufacturers are trying to integrate it into their production systems. There is a need of RMS to apply digital twin in order to hopefully improve the effectiveness and efficiency so that RPP can be automatically solved and controlled. The goal of this paper is to address the importance and requirement of the integration of digital twin simulation into RMS by providing comparison study between normal simulation program and digital twin simulation program. A case study is presented for comparison of two simulation models. Plant Simulation 11 is used for a normal simulation model, while Visual Components is used to build a digital twin simulation model.
\end{abstract}

Keywords: Reconfigurable Manufacturing System, Digital Twin, Simulation, Reconfigurable Planning Problem.

\section{Introduction}

The process of RMS requires reconfiguring machines with exact number of its components to meet the demands. Demands that periodically change also cause the machines reconfigurations. The changes of machine reconfigurations in RMS are evoked by influencing factors, to new conditions by adapting the set of machines, as known as configurations in RMS. Hence, these reconfigurations are always connected to monetary and temporal expenses. Since reconfiguration process requires high expense, effort, and time. Reflecting at those conditions, the systematic and organized planning is very necessary in RMS, whether to minimize cost or/and save time and energy. 
Therefore, the purpose of this research is firstly to introduce Reconfigurable Planning Problem (RPP) in RMS. The goal of this paper is to address the importance and requirement of the integration of digital twin simulation into RMS by providing comparison study between normal simulation program and digital twin simulation program. Since there said to be a need of simulation on digital twin to enable optimal decision-making in RMS [1]. Case study is presented for comparison study by using Plant Simulation 11 is used for normal simulation program, while Visual Components 2014 is used to build digital twin simulation program. The purpose is to find a conclusion whether digital twin is best to achieve more effective and efficient reconfigurable manufacturing system or not, as well as how to apply digital twin or other similar methodology in order to improve RMS. In this study, manufacturing process for making flip-flop is used as a case study. After experimentation of simulation model, the results will be analyzed to carry out the main objectives of the study.

\section{Reconfigurable Manufacturing System}

The reconfigurable manufacturing system (RMS) has emerged in the last few years in an attempt to achieve changeable functionality and scalable capacity [1]. Machine components, machines, cells, or material handling units can be activated, deactivated, modified, or interchanged as needed to respond quickly to changing requirements. In summary, an ideal RMS comprehends the advances of DMS and FMS [3]. RMS is marked by six core reconfigurable characteristics, as summarized below [4]:

- Customization (flexibility limited to part family)

- Convertibility (design for functionality changes)

- Scalability (design for capacity changes)

- Modularity (components are modular)

- Integrability (interfaces for rapid integration)

- Diagnosability (design for easy diagnostics)

Reconfigurable Machine Tools. Reconfigurable machine tools (RMTs) are designed for a specific range of operational requirements, and can be rapidly converted from one configuration to another. The world-first patent on RMT was issued in 1999 [2]. Fig. 1 shows an arch-type RMT that was built by the ERC-RMS and exhibited in 2002 at the International Manufacturing Show in Chicago. It was designed to drill and mill on inclined surfaces in such a way that the tool is perpendicular to the surface. 


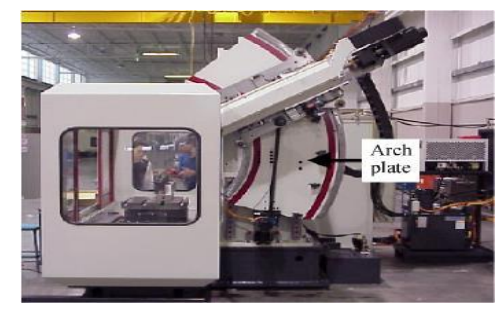

Fig. 1. Reconfigurable machine tool (RMT) developed at the ERC-RMS [4]

\section{Reconfigurable Planning Problem}

In RMS, machine components, machines, cells can be added, removed, modified, or interchanged as needed to respond quickly to changing requirements. However the process of adding or removing machines is very impractical in real life. Therefore in this study, it is assumed that system reconfiguration involves the activation and deactivation of machines components among working stations between periods, in which the representing flowchart is shown in Fig. 2. As shown in Fig.2, system throughput should be calculated first in order to determine whether it will satisfy the new demand or not. If the throughput cannot satisfy the demand, then the system requires activating additional machines. If the amount of next demand is less than the first demand, then machine/s may be deactivated since those machines will not be used to satisfy the new demand. To solve RP problems, the following notation and assumptions are used. Matlab 2013 is used to obtain the result of mathematical model.

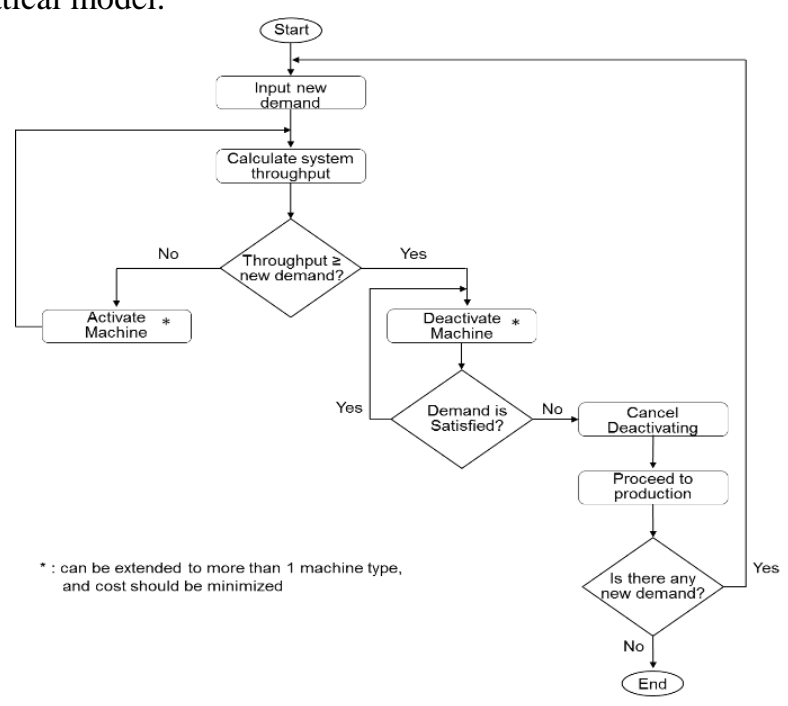

Fig. 2. Flowchart of machine reconfiguration in RMS 
Notations:

$i \quad$ Index of period $(i=1,2, \ldots, I)$

$j \quad$ Index of working station $(j=1,2, \ldots, J)$

$k \quad$ Index of machine type $(k=1,2, \ldots, K)$

$N_{i} \quad$ Minimum number of total machines in period $i$

$Q_{i} \quad$ Demands in period $i$

$T_{i} \quad$ Working time in period $i$

$T M_{i j}$ Total number of machines in working station $j$ in period $i$-1 (before activating/deactivating machines in period $i$ )

$\alpha_{k} \quad$ Activation cost of machine type $k(\$)$

$\beta_{k} \quad$ Deactivation cost of machine type $k(\$)$

$A D_{i j k}$ Number of machines type $k$ activated in working station $j$ in period $i$

$R M_{i j k}$ Number of machines type $k$ deactivated in working station $j$ in period $i$

$O_{i} \quad$ System throughput in period $i$

Firstly, we have to calculate the $N_{i}$ by dividing $Q_{i}$ by $T_{i}$, as represented in Eq.1.

$$
N_{i}=\left\lceil\frac{Q_{i}}{T_{i}}\right\rceil
$$

The objective function of reconfigurable planning is to minimize the Reconfiguration Planning cost (RP cost) which is the total summation cost of activating, deactivating, and purchasing newly bought machine, as expressed as in Eq.2.

$$
\text { Minimize } \sum_{i=1}^{I} \sum_{j=1}^{J} \sum_{k=1}^{K}\left[\left(A D_{i j k} \alpha_{k}\right)+\left(R M_{i j k} \beta_{k}\right)\right]
$$

Assumptions:

- The processing time of each operation, capacity of machine, purchasing cost of machines, and demand are known.

- Conveyors are considered as a material handling system, and sensor is installed in each machine.

- All the machines within the same stage perform exactly the same sequence of tasks

- There are reserved spaces for adding new machines in the stages and material handlers can be extended to deliver parts to the newly added machines

- Company budget for buying new machines is enough for the reserved periods.

The objective function is subject to the following constraints.

- Total number of machines must be equal or larger than the minimum number of total machines in that period.

$$
T M_{i j} \geq N_{i}
$$

- Throughput constraints must be equal or larger than the demand.

$$
O_{i} \geq \sum_{i=1}^{I} Q_{i}
$$




\section{$4 \quad$ Case Study}

In this study, RMS logic will be applied into the manufacturing process of flipflop. Sandal manufacturing is most likely labor intensive process and it cannot be fully automated. It requires craftsmanship in each phase of the production. The actual production plant assembles high quality unisex (for men and women) sandals. The size available varies from European size 36-40 (or: 235-255mm) for women and European size 40-44 (or: 255-275mm) for men. Fig. 3 shows the production sequence of two sandal products, Plain red flip-flop (R) and Couple flipflop (C).

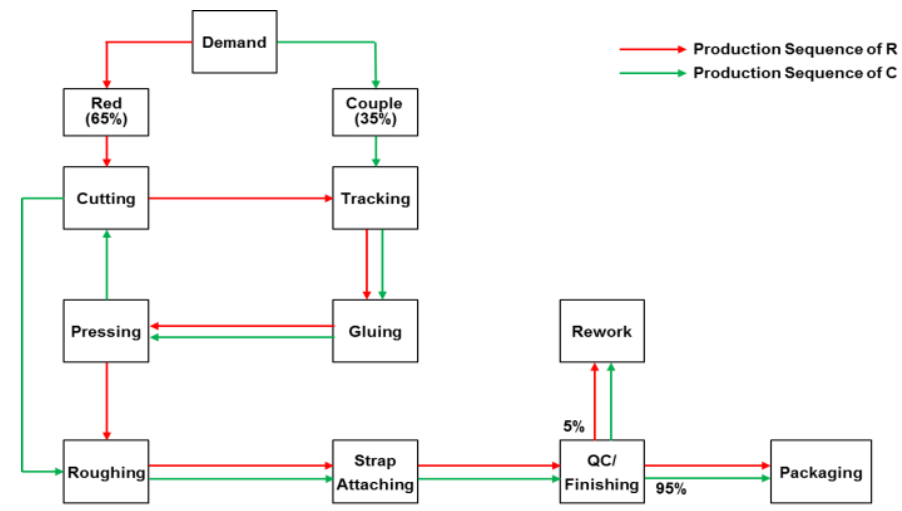

Fig. 3. Production sequence of two products

The simulation model is driven by following assumptions:

- The demand/production mix is random with exponential distribution, in terms of sandal type, size, and quantity to be produced, as shown in Table 1 . The total demand is separated into $65 \% \mathrm{R}$ and $35 \% \mathrm{C}$.

- The input buffer policy in each station for stacking trolleys.

- Every station has an input buffer where trolleys are stacked up if they cannot be processed immediately. These buffers are simulated as queues. Each coming trolley is ranked based on its order number and, then, it is released following the FIFO rule (first in - first out) when the machine is free. In this way, each station will process all trolleys with the same order number.

- Working time is 10 hours- a day.

Table 1. Demand list for 3 periods

\begin{tabular}{cccc}
\hline \multirow{2}{*}{ Period } & \multicolumn{3}{c}{ Demand (Units) } \\
\cline { 2 - 4 } & Red (65\%) & Couple (35\%) & Total \\
\hline 1 & 293 & 158 & 451 \\
2 & 59 & 33 & 92 \\
3 & 132 & 72 & 204 \\
\hline
\end{tabular}


The production strategy for the problem is make-to-order with the preorder duration of 1 day. Each day the company will receive new demand, and the company should satisfy the demand at the end of the day. The simulation is run for 1 working-month, and it is assumed that 1 month consists of 20-working days. At the end of QC work station, the result of defective and non-defective products are set to the ratio of 5:95, because the company wants to achieve $95 \%$ non-defective products. Before running the experiment, we should calculate the system throughput first, in order to set the range of demand. The value of demand should be smaller than system's maximum throughput. System throughput is the total amount of items produced by the system. By activating all machines and running the simulation, we can obtain the value of system's maximum throughput, which is 497 units per day. Based on the demand, we can calculate the total number of machines needed to satisfy the demand. For the comparison purpose, we only consider the first 3 periods only.

In Plant Simulation, every machine is built by using the provided machine/facility attributes that are already created inside the software. The machine capacity and operation time can be modified based on the requirements. Fig. 4 shows the factory layout. The advantage of using Visual Component 2014 software is that there are several machine/facility that are very close with the real life attributes. Depending on what kind of company or factory that needs to be simulated, those provided features can be very helpful. However, in order to closely compare with real data used in Plant Simulation 11, machine cycle time was modified into as close as the Plant Simulation one. Fig. 5 shows the factory layout.

Table 2 shows the processing time, and number of machines needed in Plant Simulation 11 based on the real data of company workers in Company X, while the processing time in Visual Components is already provided within the software. Assume that activation and deactivation cost for all machines are the same, activation cost is $\$ 10$ and deactivation cost is $\$ 20$. After doing the simulation on both programs for 20 days $x 1$ - hours, the result shows result summary of reconfiguration cost and number of working machine of each station is shown in Table 3. As shown in Table 3, total RP cost for Visual Components is lower than total RP cost for Plant Simulation 11.

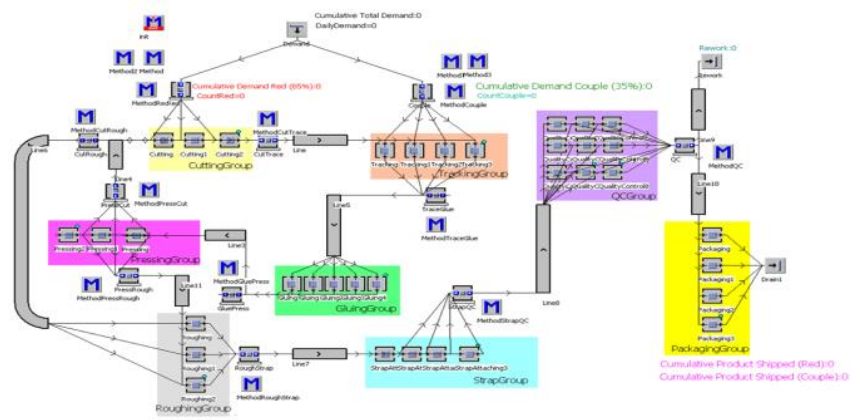

Fig. 4. Factory floor layout (Plant Simulation 11) 


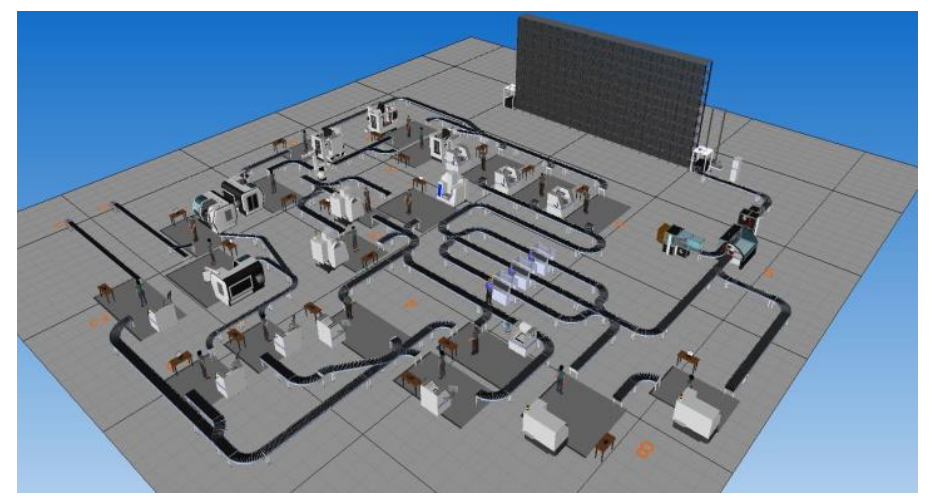

Fig. 5. Factory floor layout (Visual Components 2014)

Table 2. Processing time of work stations

\begin{tabular}{clcc}
\hline Work station $j$ & $\begin{array}{l}\text { Processing time } \\
\text { (minutes/pair) }\end{array}$ & $\begin{array}{l}\text { Number of } \\
\text { machines }\end{array}$ \\
\hline 1. & Cutting & 2 & 3 \\
2. & Pressing & 2 & 3 \\
3. $\quad$ Roughing & 2 & 3 \\
4. $\quad$ Strap attaching & 3 & 4 \\
5. $\quad$ Tracing & 3 & 4 \\
6. $\quad$ Gluing & 4 & 5 \\
7. $\quad$ QC/Finishing & 7 & 9 \\
8. & Packaging & 3 & 4 \\
\hline Total machines needed & & 35 \\
\hline
\end{tabular}

Table 3. Comparison of simulation result and reconfiguration cost

\begin{tabular}{|c|c|c|c|c|c|c|c|c|c|c|c|}
\hline \multirow{2}{*}{ Period } & \multicolumn{8}{|c|}{ Working machine in station $j$} & \multirow{2}{*}{$\begin{array}{l}\text { Number } \\
\text { of active } \\
\text { machine } \\
\text { (AD) }\end{array}$} & \multirow{2}{*}{$\begin{array}{l}\text { Number } \\
\text { of } \\
\text { inactive } \\
\text { machine } \\
\text { (RM) }\end{array}$} & \multirow{2}{*}{$\begin{array}{l}\mathrm{RP} \\
\text { Cost }\end{array}$} \\
\hline & 1 & 2 & 3 & 4 & 5 & 6 & 7 & 8 & & & \\
\hline \multicolumn{12}{|c|}{ Plant Simulation 11} \\
\hline 1 & 2 & 3 & 4 & 2 & 2 & 3 & 6 & 3 & 25 & 0 & 250 \\
\hline 2 & 1 & 1 & 1 & 1 & 1 & 1 & 2 & 1 & 0 & 16 & 320 \\
\hline 3 & 1 & 2 & 2 & 1 & 1 & 2 & 3 & 2 & 5 & 0 & 50 \\
\hline & & & & & & & & & \multicolumn{2}{|c|}{ Total RP Cost $=$} & 830 \\
\hline \multicolumn{12}{|c|}{ Visual Components 2014} \\
\hline 1 & 2 & 3 & 4 & 2 & 2 & 3 & 6 & 3 & 25 & 0 & 250 \\
\hline 2 & 1 & 1 & 1 & 1 & 1 & 1 & 2 & 1 & 0 & 16 & 320 \\
\hline 3 & 1 & 1 & 1 & 1 & 1 & 2 & 2 & 2 & 2 & 0 & 20 \\
\hline & & & & & & & & & \multicolumn{2}{|c|}{ Total RP Cost $=$} & 800 \\
\hline
\end{tabular}




\section{Conclusion}

This paper introduced a RP problem in order to anticipate any planning problem within RMS especially for the calculation of required machines to satisfy new demand, as well as presented the fact that digital twin can be and is being applied into RMS. Configurations of machines are one of the most important issues needed for an efficient and effective RMS. Machine reconfigurations are very likely occur whenever the new demands inputted into the system. Reconfiguring machines which include activating/deactivating machines needs relatively high cost. Configurations of machines are the one of the most important key issue in order to achieve an efficient and effective RMS. By applying digital twin into the RMS, the real life application seems to be possible and the system will be able to be more organized and integrated through connecting all the physical devices, operators, and data.

However, in a real production facility, additional cost factors need to be taken into account as well, such as labor, tooling, utility, floor space, operating cost, and material handlers. Since a reconfiguration process usually requires shutting down of the production system, an extra cost will occur due to the production loss during the reconfiguration process. Other constraints should also be added, such as limited factory floor space, set-up cost, and others. The future result of this study can be complied not only in RMS but also in other manufacturing concept as well.

\section{References}

1. Koren, Y., Gu, X., Guo, W.: Reconfigurable manufacturing systems: principles, design, and future trends. Front Mech Eng 2(5), 99-110 (2017).

2. Koren, Y.: General RMS Charasteristics. Comparison with Dedicated and Flexible System. In: Dashchenko A.I. (Eds.), Reconfigurable Manufacturing Systems and Transformable Factories, pp. 27-46, Springer Verlag, Berlin (2006).

3. Koren, Y., Ulsoy, A.G.: Vision, Principles and Impact of Reconfigurable Manufacturing Systems. Powertrain International 5(3), 14-21 (2002).

4. Koren, Y.: Reconfigurable Manufacturing System. CIRP Ann. Manuf. Techn 48, 527 540 (1999).

5. Wang, W., Koren, Y.: Scalability Planning For Reconfigurable Manufacturing Systems. J. Manuf. Syst 31(2), 83-91 (2012).

6. Deif, A.M., Elmaraghy, W.H.: A Systematic Design Approach for Reconfigurable Manufacturing Systems. In Advances in Design, Chapter 18, pp. 219-228. Springer, London (2006)..

7. Aboufazeli, M.: Reconfigurable Machine Tools Design Methodologies and Measuring Reconfigurability for Design Evaluation. Master Thesis, School of Industrial Engineering and Management, The Royal Institute of Technology, Sweden (2011).

8. Koren, Y., Shpitalni, M.: Design of Reconfigurable Manufacturing System. J. Manuf. Syst. 29, 130-141 (2010). 